\section{Healthcare in Low-resource Settings: the individual perspective}

\section{Norman David Goldstuck \\ Department of Obstetrics and Gynaecology, Tygerberg Hospital, Cape Town, South Africa}

A health system which does not meet the accepted norms can be called a low resource setting (LRS) for healthcare. Whose norms? Whether it be the World Health Organisation (WHO) or any other quasi governmental organisation, how does this impact the individual who needs some type of healthcare which he or she can or cannot get?

Truly personal healthcare no longer exists except in exceptional circumstances. Healthcare, like many other services in authoritarian left and right wing societies, in socialistic western societies, and even in capitalistic societies like the United States of America, is now under virtual total governmental control. This means that the individual does not ultimately decide whether he or she is in a lowresource setting, but the bureaucracy does. The central problem is that governments and organisations do not get sick (except perhaps in the metaphorical sense) and these bodies make decisions concerning those people receiving and supplying healthcare with whom they are not and will never be in direct contact. For this reason, it behoves us to look at healthcare resources from the perspective of the individual.

Healthcare resources can be grouped into the three broad categories of infrastructure, materials or supplies and human resources. While government can help bring about the first two, its ability to provide human resources (other than by way of financial inducements) is very limited. Governments are also often confused when they see the results of providing the first two and yet healthcare seems inadequate. In terms of delivering healthcare and transforming a situation from a low-resource to an adequate health resource setting providing two out of three does not prove adequate. Both government and the public at large also do not gener- ally realise that the phrase build it and they will come may apply to patients but not necessarily to healthcare practitioners.

This approach explains why patients in the US who become embroiled in the Veterans Administration or Affordable Healthcare Act problems find themselves in a high resource country which is providing them with lowresource healthcare. The same thing happens to patients in the United Kingdom, Canada and other western countries when they have to face inordinately long waiting times for surgical and other care issues.

What happens when infrastructure is poor and materials and supplies are not available but human resources (people), even relatively untrained, are? The simple answer is that no matter how low-resource the setting in terms of infrastructure and materials, concerned and compassionate human beings can always do something of value no matter how seemingly inadequate.

The conclusion here seems to be that healthcare in low-resource settings is ultimately about people and that the most precious resource available in these circumstances is not surprisingly other people. That is not to say that infrastructure and material are not very important. It is just that we must emphasize that in whatever healthcare setting, and especially in LRS, it is people helping other people that is most vital.

Articles on epidemiology and resource management as applicable to Healthcare in Lowresource Settings were originally a significant part of the mandate at the birth of this journal. ${ }^{1}$ Articles on clinical methodology and practice in the broader definition of low-resource settings as outlined would also be of interest, whether it be in relation to diagnostic, procedural or psychological aspects of healthcare practice. Particularly interesting would be how healthcare workers manage by necessarily cutting corners, i.e. omitting practises which are usually mandated by medical colleges and WHO and other guidelines but which in the circumstances become difficult or impossible to follow. Many practice guidelines presume to be evidence based but in reality still reflect the prejudices of the drafters. In LRS situations these guidelines may not even be valid or appropriate. As the world's population approaches 7 billion it will become almost impossible to provide every-
Correspondence: Norman D. Goldstuck, Department of Obstetrics and Gynaecology, Tygerberg Hospital, Green Avenue, 8001 Cape Town, South Africa.

Tel. +27.21.9384877 - Fax: +27.21.9316595

E-mail: nahumzh@yahoo.com

Key words: healthcare, low-resource settings, editorial.

Received for publication: 13 July 2014.

Accepted for publication: 13 July 2014.

This work is licensed under a Creative Commons Attribution 3.0 License (by-nc 3.0).

(C) Copyright N.D. Goldstuck, 2014

Licensee PAGEPress, Italy

Healthcare in Low-resource Settings 2014; 2:4572 doi:10.4081/hls.2014.4572

one on the planet with what is deemed to be adequate medical care. Paradoxically, as newer medications and procedures are becoming available all the time, the definition as to what constitutes adequate medical care of necessity changes. This then further changes the definition of what constitutes low-resource healthcare as high-resource healthcare becomes ever more complex and difficult. As the world's population grows, the number of individuals dragged into low-resource healthcare settings both in the developed and underdeveloped world will increase.

The solution to this problem, initially at least is to strengthen the one aspect of healthcare which can be brought into action almost immediately and that is the human resource factor. Let us focus on what individual healthcare providers at all levels e.g. doctors, nurses, medical assistants, physiotherapists, paramedics and auxiliary healthcare personnel can do to help individuals in low-resource healthcare settings.

\section{Reference}

1. Lahariya C. Introducing Healthcare in Low-resource Settings. Health Low Resour Settings 2013;1:e1. 